\title{
Extracorporeal Shockwave Therapy for Patients with Chronic Achilles Tendinopathy in Long or Short Course
}

\author{
Baili Yan $\mathbb{D}^{1},{ }^{1}$ Yuan Wan $\mathbb{D}^{1},{ }^{1}$ Hong Zhang $\mathbb{D},{ }^{1}$ MengTing Pan $\mathbb{D}^{1},{ }^{1}$ and Cheng Zhou $\mathbb{D}^{2}$ \\ ${ }^{1}$ Department of Pain Management, Wuhan No.1 Hospital, Wuhan, China \\ ${ }^{2}$ Department of Hepatobiliary Surgery, Wuhan No.1 Hospital, Wuhan, China
}

Correspondence should be addressed to Cheng Zhou; cheng.zhou@whyyy.com

Received 12 April 2020; Accepted 28 July 2020; Published 12 August 2020

Academic Editor: George Babis

Copyright ( 2020 BaiLi Yan et al. This is an open access article distributed under the Creative Commons Attribution License, which permits unrestricted use, distribution, and reproduction in any medium, provided the original work is properly cited.

\begin{abstract}
Object. In this study, our aim is to evaluate the efficiency of extracorporeal shockwave therapy (ESWT) on chronic Achilles tendinopathy (CAT) with different courses of disease. Methods. The data of 66 patients with CAT received ESWT was reviewed. According to the disease courses, those cases were allocated to short-term group (ST group, symptom duration 3-6 months) and long-term group (LT group, symptom duration $>6$ months). Propensity scores match (PSM) method was conducted to eliminate the confound factors in baseline features including gender, sport history, sides, type of CAT, BMI (body mass index), age, and scores evaluated by AOFAS (American Orthopedic Foot and Ankle Society) and VAS (Visual Analogue Scale) before ESWT. After balancing the features between ST and LT group, postinterventional VAS, AOFAS, and rate of Likert satisfaction scale at the 3rd month after first ESWT was statistically analyzed. Results. Among the baseline features in ST and LT group, gender (female ratio, $44.4 \%$ vs $71.4 \%, p=0.041)$ and BMI $(23.26 \pm 2.15$ vs $24.63 \pm 2.41, p=0.024)$ were identified as confound factors. After elimination of biased features with PSM, 3 months after first ESWT, AOFAS and VAS in both groups are significantly improved, when compared with their scores at baseline $(p<0.01)$. Moreover, at postintervention month 3 (PIM3), AOFAS in ST group is significantly higher than LT group ( $85.08 \pm 9.83$ vs $76.76 \pm 9.85, t=76.76 \pm 9.85, p=0.019)$, and the rate of Likert satisfaction in ST group is better than LT group; although, it did not reach but close to significant level $(70.6 \%$ vs $\left.47.1 \%, \chi^{2}=1.943, p=0.163\right)$. However, there is no statistical difference of VAS scores between two groups after ESWT $(1.96 \pm 0.98$ vs $2.24 \pm 1.29, t=0.703, p=0.487)$. Conclusions. ESWT could effectively relieve pain and improve function of hind foot in patients with chronic Achilles tendinopathy, and especially, it could offer better benefit on functional improvement in patients with short duration of CAT symptom.
\end{abstract}

\section{Introduction}

Chronic Achilles tendinopathy (CAT) is a common pain disease and prevails in athletes, middle-aged male runners, and sedentary population [1]. In China, with the enhancement of awareness of sports and health, the number of physical activity participants is rising; according to the report from Chen et al. [2], the percentage of daily exercisers in Chinese urban resident has reached $19.57 \%$, unfortunately, of which, CAT occurs more frequently in running population, for about 5$18 \%[3,4]$ of incidence. Typical symptom of CAT composes of Achilles pain, swell, and functional limitation. CAT is complicated in its pathogenesis, which is not fully elucidated yet. Conservative therapies, such as immobilization, nonste- roid anti-inflammation agents, Laser therapy, hyaluronan (HA) or platelet-rich plasma injections, and eccentric exercises are all regarded as treatments in the first line.

However, the extracorporeal shockwave therapy (ESWT), first proposed in management of CAT by Furia [5], has advantages of safety, minimal invasive, versatile, economic, which makes it become gradually increasing of application in clinical practice, and an important supplement for conservative therapy as well. However, in Stania et al.'s [6] meta report, by analyzing of 22 clinical trials, he believed that because of complexity of biologic reaction results from CAT, great diversity among different ESWT algorithms, and lack of objective parameters for outcome measurements, the value of ESWT in CAT therapy is not fully evaluated. In addition, 
there is also an opinion that indicates longer duration of CAT directly correlated to insufficient therapeutic effects offered by ESWT [7], but to the best of our knowledge, there is no literature concerning the relationship between the CAT duration and ESWT efficacy. For aforementioned reasons, we tried to explore if early ESWT intervention is a better approach for the recovery of patient with Achilles tendinopathy. The data of patients received ESWT because of CAT in our institute was collected and analyzed, retrospectively, and reported as follows:

\section{Material and Methods}

The medical records of patients suffered from CAT and treated with ESWT at outpatient or inpatient of pain management department in our institute, from Dec. 2017 to Dec. 2019, were collected retrospectively. There are 66 cases in total. Diagnostic standard of Achilles tendinopathy is defined as having pain and tenderness located on unilateral or bilateral Achilles area. Inclusion criterion is the patients clinically diagnosed as CAT with or without radiology evidence, which had a symptom persisted at least 3 months. Exclusion criteria were settled as patients who administrated with nonsteroid anti-inflammation agent within one week before ESWT or received steroid therapy or previous ESWT within 3 months before ESWT, or concomitant with anatomic foot malformation, fracture, or operation history of foot and heel. Ethical approval is not needed because of the retrospective natural in our study.

2.1. Case Grouping. According to the duration of CAT symptom, 66 patients were divided into long-term group (LT) for 21 cases, symptom persisting longer than 6 months; shortterm group (ST) for 45 cases, duration from 3 to 6 months.

2.2. Type of CAT. Insertional type defined as pain located at $2 \mathrm{~cm}$ within the insertion of tendon into the calcaneus. Noninsertional type is defined as pain located $2-6 \mathrm{~cm}$ proximal to calcaneal insertion point [8].

2.3. Functional Parameter of Foot and Ankle. Scores system developed by American Orthopedic Foot and Ankle Society (AOFAS) was applied in our study [9]. 100 points were designed in AOFAS system in total, in which 40 points stand for pain severity, 50 points present function state, and 10 points for alignment.

2.4. Pain Parameter. Visual Analogue Scale (VAS) was performed [10]. From 0 to 10 points $(10$ points $=$ serious pain, $0=$ painless), patients were asked to decide the scores based on their subjective perception of pain severity.

2.5. Satisfactory Rate Parameter. 6-points Likert satisfaction scale was used in our study (Table 1). Satisfaction was defined as 1-4 points fed back by patients, unsatisfaction was regarded as scores from 5 to 6 points, and rate was calculated for further analyze.

2.6. ESWT Procedure. Equipment we used in our study is a radial extracorporeal shockwave instrument (Swiss DolorClast Smart20, EMS Elctro Medical Systems, Munich,
TABLE 1: 6-points Likert satisfaction score.

\begin{tabular}{ll}
\hline Level of improvement & 1 \\
\hline Completely recovered & 2 \\
Much improved & 3 \\
Somewhat improved & 4 \\
Hardly improved & 5 \\
Not improved & 6 \\
Worse
\end{tabular}

Germany). In each therapy episode, frequency was set as $4 \mathrm{~Hz}$ or $8 \mathrm{~Hz}$ and 2000 pulse, and $0.096-0.137 \mathrm{~mJ} / \mathrm{mm}^{2}$ was given. Patients took on prone position, and the most tenderness area complained by patients was treated as target region. ESWT manipulators were specialized physiotherapists in our department. During procedures, therapists exert pressure depending on patient's maximal tolerance of pain. 3-5 sessions of ESWTs were given at weekly interval without any additional exercises. Except for diclofenac diethylamine emulgel (Novartis China, Beijing) was used as lubricator before intervention; no other analgesic agent was given. Patients were educated to avoid vigorous physical activities during treatment session.

2.7. Follow-Ups and Parameters. In all of 66 cases, the data regarding gender, sport history, sides (superiority or nonsuperiority), type (insertion or no insertion), BMI (body mass index), age, AOFAS scores, and VAS scores evaluated before first ESWT were collected as baseline features. Patients were routinely followed up at outpatient at the 3rd month after the first ESWT, the scores of AOFAS, VAS, and Likert satisfaction were reevaluated, which were collected as outcome parameters for statistical analysis (see Figure 1). Adverse events after ESWT was also recorded.

2.8. Statistical Analysis. Continuous variables were tested with Kolmogorov-Smirnov for normality of distribution first and then compared between LT and ST groups by Student's $t$ -test. The variance of categorical variables was determined with chi-square test. To minimize the bias between LT and ST groups at the baseline, we performed $1: 1$ propensity score match (PSM) with the nearest neighbor matching algorithm and set parameter of match tolerance as 0.1 . After match, the variance derived from intergroups and intragroups was further analyzed with two tails Student's $t$-test in quantity data, and by chi-square or Fisher's exact test in quality data. We used SPSS 24.0 (SPSS Inc., Chicago, Illinois, USA) for all statistical analysis, and $p<0.05$ suggested a statistically significant difference.

\section{Results}

First, we compared factors including gender, sport history, sides, type, BMI, age, and preinterventional AOFAS and VAS scores between ST and LT groups, to identify the bias characteristics at baseline (Table 2). Among enrolled 66 cases 


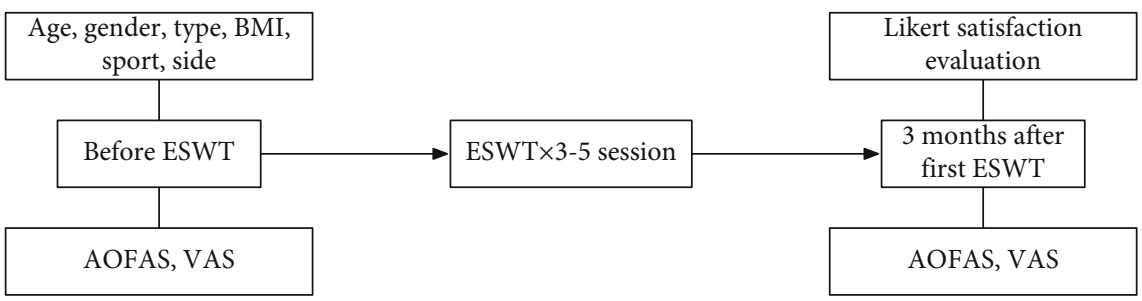

FIgURE 1: Flow-chart of parameter evaluation and follow-up.

Table 2: Baseline features in patients with chronic Achilles tendinopathy before PSM.

\begin{tabular}{lcccc}
\hline Characteristics & $\begin{array}{c}\text { ST group } \\
(n=45)\end{array}$ & $\begin{array}{c}\text { LT group } \\
(n=21)\end{array}$ & $\mathrm{t}\left(\chi^{2}\right)$ & $p$ \\
\hline Gender $[n(\%)]$ & & & & \\
$\quad$ Male & $25(55.6)$ & $6(29.6)$ & 4.186 & $0.041^{*}$ \\
$\quad$ Female & $20(44.4)$ & $15(71.4)$ & & \\
Sports [n(\%)] & & & & \\
$\quad$ Yes & $28(62.2)$ & $10(47.6)$ & 1.25 & 0.264 \\
$\quad$ No & $17(37.8)$ & $11(52.4)$ & & \\
Sides [n(\%)] & & & & \\
$\quad$ Superiority & $26(57.8)$ & $12(57.1)$ & 0.002 & 0.961 \\
& $19(42.2)$ & $9(42.9)$ & & \\
Nonsuperiority & & & & \\
Type $[n(\%)]$ & $17(37.8)$ & $7(33.3)$ & 0.122 & 0.727 \\
Insertion & $28(62.2)$ & $14(66.7)$ & & \\
& & & & \\
Noninsertion & $23.26 \pm 2.15$ & $24.63 \pm 2.41$ & -2.317 & $0.024^{*}$ \\
BMI & $56.77 \pm 8.68$ & $60.80 \pm 8.74$ & -1.754 & 0.084 \\
Age $(y)$ & $63.88 \pm 10.44$ & $63.06 \pm 11.52$ & 0.288 & 0.774 \\
AOFAS & $6.03 \pm 1.09$ & $5.61 \pm 1.13$ & 1.431 & 0.157 \\
VAS & & & &
\end{tabular}

${ }^{*}$ Compared with ST group, the difference is significant, $p<0.05$.

of CAT, $68.18 \%$ was in ST group $(n=45)$, and $31.81 \%$ in LT group $(n=21)$.

Based on baseline characteristics before match, regarding to gender ratio, CAT influenced significantly more female patients in LT group than ST group $(71.4 \%$ vs $44.4 \%, p<0.05)$; meanwhile, BMI was also significantly higher in LT group $(24.63 \pm 2.41$ vs $23.26 \pm 2.15, p<0.05)$. In terms of age factor, patients in LT group is elder when compared with ST group; although, the difference did not reach, but very close to significant level $(60.80 \pm 8.74$ vs $56.77 \pm 8.68, p=0.084)$. The variances among other features are all insignificant.

After balanced with 1:1 propensity score matching, the data of 34 patients was preserved, and 17 cases for each group. 8 baseline characteristics and 3 outcome parameters, including AOFAS, VAS, and Likert satisfaction rate at the 3rd month after first ESWT, were further compared and analyzed. See Table 3.

After matching, the female ratio at baseline changed to $64 \%$ in ST group and $76.5 \%$ in LT group; no significant dif- ference was identified anymore, $p=0.45$. Moreover, the bias of BMI between two groups is no longer existing, when compared ST group $(23.96 \pm 1.93)$ again with LT group $(24.11 \pm 2.25), p=0.831$. Between two groups, the balance of age factor remains unchanged $(59.91 \pm 9.62$ vs. $61.34 \pm$ 9.33, $p=0.662$ ).

After PSM, we compared AOFAS and VAS scores of both groups with their baseline counterpart and also check the difference on postinterventional AOFAS, VAS, and Likert satisfaction rate between ST and LT group (Figures 2 and 3).

After PSM, at the 3rd month after first ESWT, compared AOFAS scores between two groups, ST group (85.08 \pm 9.83$)$ is significantly higher than LT group $(76.76 \pm 9.85)$, and $p$ value was 0.019 ; however, there is no significant difference of VAS scores was observed $(1.96 \pm 0.98$ vs 2.24 $\pm 1.29, p=0.487$ ) between two groups. Compared with baseline, postinterventional AOFAS scores in ST group is significantly increased $(65.43 \pm 10.05$ vs $85.08 \pm 9.83$, $p<0.01)$, and VAS scores reduced significantly $(5.92 \pm 1.12$ vs $1.96 \pm 0.98, p<0.01)$. The similar tendency of periinterventional changes of AOFAS (from $62.80 \pm 11.90$ to $76.76 \pm 9.85, p<0.01$ ) and VAS (from $5.87 \pm 1.10$ to 2.24 $\pm 1.29, p<0.01$ ) was seen in LT group.

At the 3rd month after the first ESWT, the satisfaction rate was compared between two groups (Figure 4).

Three months after the first session of ESWT, satisfaction rate of ST group got to $70.6 \%(12 / 17)$, which is higher than that of LT group $(47.1 \%, 8 / 17)$. Although the difference did not reach, but is very close to significant level $(p=0.163)$.

3.1. Adverse Events. In most of our cases, ecchymosis and numbness were observed and relieved later without any interventions. No bleeding or tendon rupture was reported.

\section{Discussion}

Our results show that ESWT could effectively relieve pain and improve the function of hind foot in CAT patients. Especially, on the aspect of functional improvement, such positive effect is more profound in short course cases. In fact, not only the pathogenesis of CAT is not fully understood [11] but also the therapeutic mechanism of ESWT related to CAT remains not well elucidated [3]. Therefore, better results achieved in short course patients might be closely involved with its pathogenesis and therapeutic mechanism and are worth to be explored.

Epidemiologically, in term of gender tendency, some authors believe that CAT influences more male patients than 
TABLE 3: Baseline features and outcome parameters in patients with chronic Achilles tendinopathy after PSM.

\begin{tabular}{|c|c|c|c|c|}
\hline Characteristics & ST group $(n=17)$ & LT group $(n=17)$ & $\mathrm{t}\left(\chi^{2}\right)$ & $p$ \\
\hline \multicolumn{5}{|l|}{ Gender $[n(\%)]$} \\
\hline Male & $6(35.3)$ & $4(23.5)$ & 0.567 & 0.452 \\
\hline Female & $11(64.7)$ & $13(76.5)$ & & \\
\hline \multicolumn{5}{|l|}{ Sports $[n(\%)]$} \\
\hline Yes & $12(70.6)$ & $8(47.1)$ & 1.943 & 0.163 \\
\hline No & $5(29.4)$ & $9(52.9)$ & & \\
\hline \multicolumn{5}{|l|}{ Sides $[n(\%)]$} \\
\hline Superiority & $10(58.8)$ & $10(58.8)$ & 0 & 1 \\
\hline Nonsuperiority & $7(41.2)$ & $7(41.2)$ & & \\
\hline \multicolumn{5}{|l|}{ Type $[n(\%)]$} \\
\hline Insertion & $6(35.3)$ & $7(41.2)$ & 0.124542 & 0.724 \\
\hline Noninsertion & $11(64.7)$ & $10(58.8)$ & & \\
\hline BMI & $23.96 \pm 1.93$ & $24.11 \pm 2.25$ & 0.215 & 0.831 \\
\hline Age $(y)$ & $59.91 \pm 9.62$ & $61.34 \pm 9.33$ & 0.441 & 0.662 \\
\hline \multicolumn{5}{|l|}{ Preintervention } \\
\hline AOFAS & $65.43 \pm 10.05$ & $62.80 \pm 11.90$ & 0.695 & 0.492 \\
\hline VAS & $5.92 \pm 1.12$ & $5.87 \pm 1.10$ & 0.127 & 0.9 \\
\hline \multicolumn{5}{|l|}{ Postintervention } \\
\hline AOFAS & $85.08 \pm 9.83^{\Delta \Delta}$ & $76.76 \pm 9.85^{\Delta \Delta}$ & 2.464 & $0.019^{* \Delta}$ \\
\hline VAS & $1.96 \pm 0.98^{\Delta \Delta}$ & $2.24 \pm 1.29^{\Delta \Delta}$ & 0.703 & 0.487 \\
\hline Sat. rate $[n(\%)]$ & $12(70.6)$ & $8(47.1)$ & 1.943 & 0.163 \\
\hline
\end{tabular}

${ }^{*}$ Compared with ST group in same period, the difference is significant, and $p<0.05 ;{ }^{\Delta \Delta}$ compared with preintervention in same group, the difference is significant, and $p<0.01$.

AOFAS scores before and after ESWT

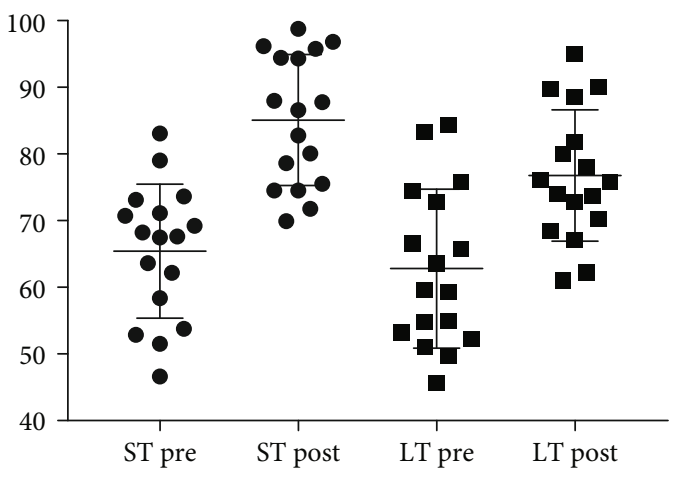

FIgURE 2: AOFAS scores before and after ESWT.

the female [12], which possibly be related to more frequent physical activities in male exerciser. In fact, a slightly higher incidence of female patients, about 53-59\% [13-19], was reported in the majority of literatures, even as high as $77.4 \%$ or $81.8 \%[11,20]$ was mentioned. In our study, the general rate of female patient is $53.3 \%$ (35/66) and slightly higher than male, which is consist with most of articles. Taylor et al. [21] recruited only refractory CAT patients with average course from 24 to 42 months in their trial. He reported a low prevalence rate in female with longer persistence of symptom. But the rate of female patient with long course is significantly higher than those with short course in our study, which is not only contrary to Taylor's result but also lead to biased character at baseline. Therefore, we believe that if there is any gender tendency in the occurrence of Achilles tendinopathy is still an epidemiological question needs to be explored.

The other bias factor at baseline is BMI. To date, the threshold for overweight in the Chinese population is still 24, which reflects the background of nonuniversality of excessive body weight in China. However, with the change of diet pattern, BMI tends to gradually increase in the Chinese population. In a cross-sectional observational study with large samples, Zhang et al. [22] reported BMI had reached $25.6 \pm 3.1$ in Chinese male and $23.4 \pm 3.2$ in the female. Similarly, in our long-term group, BMI was $24.11 \pm$ 2.25, not only greater than in short-term group $(23.26 \pm 2.15)$ but also beyond the Chinese threshold of overweight. Although, there is point of view proposes that weight is also an intrinsic fact responsible for CAT [6]; in fact, few literatures focused on BMI level in patients with CAT. In their trials of Achilles tendinopathy, Pinitkwamdee et al. [11] and Mansur et al. [23] described BMI could be as high as 28.2 and 29.2, almost reach the obese standard in the western. Etiologically, developing of CAT possibly because of ankle articular overuse and accumulation of minimal trauma on it [24]. Overweight will cause increased and persisting stress on Achilles's tendon through daily activities; likewise, it could be a possible reason of biased BMI in our longterm group as well. 
VAS scores before and after ESWT

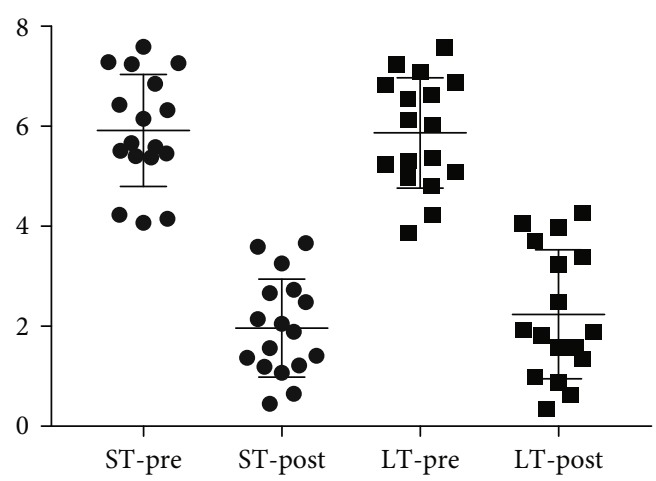

Figure 3: VAS scores before and after ESWT.

Satisfactory percentage after ESWT

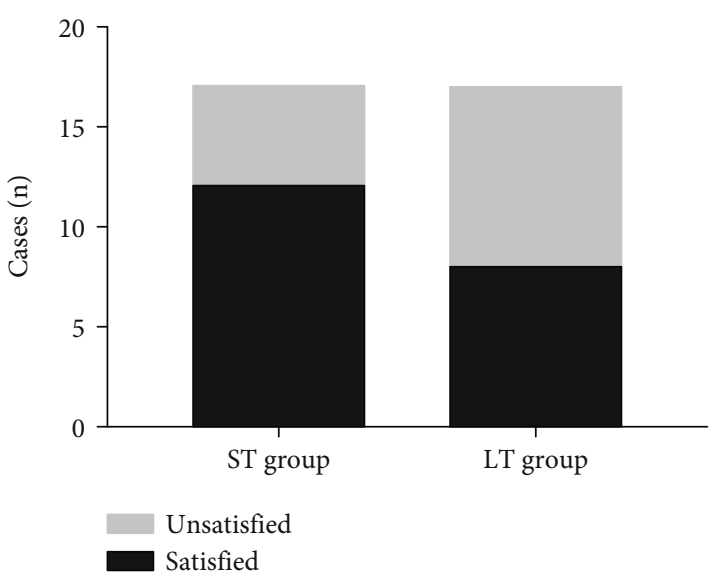

FIGURE 4: Likert Satisfaction percentage after ESWT.

In the view of therapeutic mechanism, via mechanic stimulation, ESWT could promote the expression of inflammation factors, enhance the proliferation of tenocyte and synthesis of collagen, in turn, repair damaged tendinous tissue and improve the function of Achilles's tendon [25]. Meanwhile, the shock wave could also benefit on the reduction of local substance P [26], destruction of unmyelinated nerve fibres [27], consequently relieve the pain result from CAT. Therefore, pain relief and functional improvement are the most important therapeutic effects. In a systematic review by Al-Abbad and Simon [3], the efficiency of ESWT in pain relief had been consistently proven in 4 trails, and so as functional improvement in 5 trails. In our study, three months after the first shock wave therapy, both AOFAS and VAS scores were improved significantly, which is coincident with most of literatures. Njawaya et al. [19] reported that, with or without guidance of ultrasound, 3 months after ESWT, the converted VAS score is around 2 points, which is very close to our general VAS score $(1.92 \pm 1.10)$ measured at corresponding PIM. A placebo-controlled, prospective research from Rasmussen et al. [18] shows ESWT could raise AOFAS to 81.0 at the $3 \mathrm{rd}$ month after treatment. Our result manifests an average AOFAS score of $80.49 \pm 10.4$ in general, which is very similar. Therefore, with our retrospective data, we once more proved the therapeutic effects of ESWT on patients with chronic Achilles tendinopathy overall; no matter the disease course is long or short.

Although, not all the studies took duration of symptom as baseline parameters, but most of authors chose symptom lasting more than 6 months as inclusion criteria, which in turn, make most of patients treated with ESWT, is actually with long disease course. In Taylor et al.'s [21] report, both insertion and noninsertion groups were of long course. 16 weeks after shock wave therapy, the activity VAS score reached only 4.4 and 4 in their groups, respectively. The average course of CAT is 18.23 months in the series recruited by Wheeler et al. [17], and he described the VAS scored only 4.35 at PIM3. In addition, Notarnicola et al. [14] enrolled patients with persisting symptom more than 6 months; 2 months after ESWT, VAS is still $5.4 \pm 2.7$. On the contrary, Pinitkwamdee et al. [11] took a patient with average disease course of 4.5 months for observation; 16 weeks after ESWT, VAS scores were reduced to $3.00 \pm 2.15$. Intergroup data were compared after PSM in our study, though VAS score is lower in ST group, but difference is insignificant. The tendency regarding the relationship between longer course and insufficient relief of pain demonstrated in above-listed literatures is not consistently discovered by our study. Leong et al. [28] treated Achilles's tendon of health adults with ESWT, and results showed shock wave could only enhance immediate pressure pain threshold, which restored to normal only 3 hours after intervention. His evidence suggests analgesic effect of ESWT is temporal and transient. Essentially, the quickness of pain relief depends on the speed of neural selfrepair. In general, the length of course is a proxy for refractory of certain disease, whether there is a special neurological mechanism underlying the phenomena, in which the pain caused by CAT is hard to be alleviated by short-term shock wave, is a problem need to be investigated in experiment research in the future.

AOFAS is a valuable indicator reflects the function of hind foot, which was often adopted as an outcome parameter in literatures. In Carulli et al.'s [16] series, the average length of disease term is 6.7 months, which is not so prominent in duration. But 6 months after ESWT, AOFAS scores were only improved to $77 \pm 2.4$. Notranicola et al. [14] recruited patients with symptom duration over 6 months, and 2 months after shock wave therapy, AOFAS raised to only 73 \pm 22.9 . On the contrary, Vahdatpour et al. [20] observed patients of 4.5 months in disease duration; AOFAS reached as high as $85.85 \pm 7.8$ at 16 weeks after ESWT. Similarly, Pavone et al. [29] enrolled patients with disease course over 3 months for research, combined with eccentric exercise, AOFAS scores were achieved as $85.2 \pm 4.1$. As to our ST group, 3 months after the first ESWT, the score of AOFAS went up to $85.08 \pm 9.83$, which is very similar to the value described by latter two authors, and significantly better than LT group (76.76 \pm 9.85$)$. In scoring system of AOFAS, 40 points stand for pain; in other words, only the remaining 50 points of function explain all the changes of AOFAS. In a sense, instead of analgesic effect, ESWT is more powerful in function improvement. The shock wave could transform 
mechanical stimuli into biological signal which upregulates proliferation of fibroblast, increases genetic expression of TGF-beta1 (transforming growth factor beta-1), collagen I and III, consequently promotes the release of TGF-beta1, synthesis of collagen, and enhances the restoration of tendon as result [30]. Tendon repair is a gradual healing process obviously associated with severity of tissue damage. In most situations, patients with shorter duration are mild in severity, thus are more prone to be benefited by ESWT.

This study has several limitations. As a review study adopting statistical method of propensity score match, theoretically, it has similar evidential value as in prospective studies, but the research is fundamentally retrospective, bias factors might be missed during data collection, will inevitably never be balanced by PSM at baseline, thus limit the power of our study. Secondly, samples are small in our study, some were further lost during match process. Insufficient quantities in sample probably lead to biased conclusion. Therefore, we believe it will be more persuasive to validate our conclusion in prospective, randomized, controlled trials with large sample.

In conclusion, our retrospective study found ESWT could effectively relieve pain and improve impaired function caused by chronic Achilles's tendonipathy. It offers better functional amelioration of hind foot in the patients with short disease course.

\section{Abbreviations}

BMI: $\quad$ Body mass index

CAT: $\quad$ Chronic Achilles tendinopathy

ESWT: $\quad$ Extracorporeal shockwave therapy

HA: Hyaluronan

PIM: $\quad$ Postinterventional month

PSM: $\quad$ Propensity scores match

TGF-beta1: Transforming growth factor beta-1

VAS: Visual Analogue Scale.

\section{Data Availability}

The data used to support the findings of this study are available from the corresponding author upon request.

\section{Conflicts of Interest}

The author(s) declare(s) that they have no conflicts of interest.

\section{Authors' Contributions}

The first author is BaiLi Yan, and the first coauthor is Yuan Wan.

\section{References}

[1] A. C. Egger and M. J. Berkowitz, "Achilles tendon injuries," Current Reviews in Musculoskeletal Medicine., vol. 10, no. 1, pp. 72-80, 2017.

[2] D. R. Chen and Y. C. Lin, "Social identity, perceived urban neighborhood quality, and physical inactivity- A comparison study of China, Taiwan, and South Korea," Health \& Place, vol. 41, pp. 1-10, 2016.

[3] H. Al-Abbad and J. V. Simon, "The effectiveness of extracorporeal shock wave therapy on chronic achilles tendinopathy: a systematic review," Foot \& Ankle International, vol. 34, no. 1, pp. 33-41, 2013.

[4] M. Krishna Sayana and N. Maffulli, "Insertional Achilles tendinopathy," Foot and Ankle Clinics, vol. 10, no. 2, pp. 309320, 2005.

[5] J. P. Furia, "High-energy extracorporeal shock wave therapy as a treatment for insertional Achilles tendinopathy," The American Journal of Sports Medicine, vol. 34, no. 5, pp. 733-740, 2017.

[6] M. Stania, G. Juras, D. Chmielewska, A. Polak, C. Kucio, and P. Król, "Extracorporeal shock wave therapy for Achilles tendinopathy," BioMed Research International, vol. 2019, Article ID 3086910, 13 pages, 2019.

[7] J. D. Rompe, B. Nafe, J. P. Furia, and N. Maffulli, "Eccentric loading, shock-wave treatment, or a wait-and-see policy for tendinopathy of the main body of tendo Achillis: a randomized controlled trial," The American Journal of Sports Medicine, vol. 35, no. 3, pp. 374-378, 2017.

[8] C. R. Carcia, R. L. Martin, and D. K. Wukich, "Achilles pain, stiffness, and muscle power deficits: Achilles tendinitis," Journal of Orthopaedic \& Sports Physical Therapy, vol. 40, no. 9, pp. A1-A26, 2010.

[9] H. B. Kitaoka, I. J. Alexander, R. S. Adelaar et al., "Clinical rating systems for the ankle-hindfoot, midfoot, hallux, and lesser toes," Foot \& Ankle International, vol. 18, no. 3, pp. 187-188, 1997.

[10] P. S. Myles, S. Troedel, M. Boquest, and M. Reeves, “The pain visual analog scale: is it linear or nonlinear?," Anesthesia and Analgesia, vol. 89, no. 6, pp. 1517-1520, 1999.

[11] S. Pinitkwamdee, S. Laohajaroensombat, J. Orapin, and P. Woratanarat, "Effectiveness of extracorporeal shockwave therapy in the treatment of chronic insertional Achilles tendinopathy," Foot \& Ankle International, vol. 10, 2020.

[12] A. Scott and M. Ashe, "Common tendinopathies in the upper and lower extremities," Current Sports Medicine Reports, vol. 5, no. 5, pp. 233-241, 2006.

[13] N. Lynen, T. De Vroey, I. Spiegel, F. Van Ongeval, N. J. Hendrickx, and G. Stassijns, "Comparison of peritendinous hyaluronan injections versus extracorporeal shock wave therapy in the treatment of painful Achilles' tendinopathy: a randomized clinical efficacy and safety study," Archives of Physical Medicine and Rehabilitation, vol. 98, no. 1, pp. 64-71, 2017.

[14] A. Notarnicola, G. Maccagnano, S. Tafuri, M. I. Forcignanò, A. Panella, and B. Moretti, "CHELT therapy in the treatment of chronic insertional Achilles tendinopathy," Lasers in Medical Science, vol. 29, no. 3, pp. 1217-1225, 2014.

[15] J. D. Rompe, J. Furia, and N. Maffulli, "Eccentric loading versus eccentric loading plus shock-wave treatment for midportion Achilles tendinopathy," The American Journal of Sports Medicine, vol. 37, no. 3, pp. 463-470, 2017.

[16] C. Carulli, F. Tonelli, M. Innocenti, B. Gambardella, F. Muncibì, and M. Innocenti, "Effectiveness of extracorporeal shockwave therapy in three major tendon diseases," Journal of Orthopaedics and Traumatology, vol. 17, no. 1, pp. 15-20, 2016.

[17] P. C. Wheeler and C. Tattersall, "Novel Interventions for Recalcitrant Achilles Tendinopathy: Benefits Seen Following 
High-Volume Image-Guided Injection or Extracorporeal Shockwave Therapy-A Prospective Cohort Study," Clinical Journal of Sport Medicine, vol. 30, no. 1, pp. 14-19, 2020.

[18] S. Rasmussen, M. Christensen, I. Mathiesen, and O. Simonsen, "Shock wave therapy for chronic Achilles tendon pain: a randomized placebo-controlled trial," Clinical Orthopaedics and Related Research, vol. 440, pp. 199-204, 2005.

[19] M. M. Njawaya, B. Moses, D. Martens et al., "Ultrasound guidance does not improve the results of shock wave for plantar fasciitis or calcific Achilles tendinopathy: a randomized control trial," Clinical Journal of Sport Medicine, vol. 28, no. 1, pp. 21-27, 2018.

[20] B. Vahdatpour, H. Forouzan, F. Momeni, M. Ahmadi, and P. Taheri, "Effectiveness of extracorporeal shockwave therapy for chronic Achilles tendinopathy a randomized clinical trial," Journal of Research in Medical Sciences : The Official Journal of Isfahan University of Medical Sciences, vol. 23, p. 37, 2018.

[21] J. Taylor, S. Dunkerley, D. Silver et al., "Extracorporeal shockwave therapy (ESWT) for refractory Achilles tendinopathy- a prospective audit with 2-year follow up," The Foot, vol. 26, pp. 23-29, 2016.

[22] R. Zhang, S.-Y. Dong, F. Wang et al., “Associations between body composition indices and metabolic disorders in Chinese adults," Chinese Medical Journal, vol. 131, no. 4, pp. 379-388, 2018.

[23] N. S. B. Mansur, T. Baumfeld, F. Villalon et al., "Shockwave therapy associated with eccentric strengthening for Achilles insertional tendinopathy-a prospective study," Foot \& Ankle Specialist, vol. 12, no. 6, pp. 540-545, 2019.

[24] B. R. Freedman, J. A. Gordon, and L. J. Soslowsky, “The Achilles tendon: fundamental properties and mechanisms governing healing," Muscles, Ligaments and Tendons Journal, vol. 4, no. 2, pp. 245-255, 2014.

[25] X. J. Duan, L. Yang, and H. Q. Huang, "Progress of extracorporeal shock wave therapy in chronic achilles tendinopahty Chinese," Journal of the Frontiers of Medical Science, vol. 7, no. 11, pp. 5-9, 2015.

[26] J. Hausdorf, M. A. M. Lemmens, S. Kaplan et al., "Extracorporeal shockwave application to the distal femur of rabbits diminishes the number of neurons immunoreactive for substance P in dorsal root ganglia L5," Brain Research, vol. 1207, pp. 96-101, 2008.

[27] S. Ohtori, G. Inoue, C. Mannoji et al., "Shock wave application to rat skin induces degeneration and reinnervation of sensory nerve fibres," Neuroscience Letters, vol. 315, no. 1-2, pp. 5760, 2001.

[28] H. T. Leong, S. Docking, M. Girdwood, C. Bonello, J. Cook, and E. Rio, "Extracorporeal shock wave therapy immediately affects Achilles tendon structure and widespread pressure pain thresholds in healthy people," American Journal of Physical Medicine \& Rehabilitation, vol. 98, no. 9, pp. 806-810, 2019.

[29] V. Pavone, L. Cannavò, A. Di Stefano, G. Testa, L. Costarella, and G. Sessa, "Low-energy extracorporeal shock-wave therapy in the treatment of chronic insertional Achilles tendinopathy: A case series," BioMed Research International, vol. 2016, Article ID 7123769, 4 pages, 2016.

[30] Y. H. Chao, Y. H. Tsuang, J. S. Sun et al., "Effects of shock waves on tenocyte proliferation and extracellular matrix metabolism," Ultrasound in Medicine \& Biology, vol. 34, no. 5, pp. 841-852, 2008. 